Annals of Traditional Chinese Medicine-Vol. 3

\title{
ALTERNATIVE TREATMENT for
}



Editors

Ping-Chung Leung

The Chinese University of Hong Kong

Harry Fong

University of Illinois at Chicago, USA 


\title{
Published by
}

World Scientific Publishing Co. Pte. Ltd.

5 Toh Tuck Link, Singapore 596224

USA office: 27 Warren Street, Suite 401-402, Hackensack, NJ 07601

UK office: 57 Shelton Street, Covent Garden, London WC2H 9HE

\section{British Library Cataloguing-in-Publication Data}

A catalogue record for this book is available from the British Library.

\begin{abstract}
Annals of Traditional Chinese Medicine - Vol. 3 ALTERNATIVE TREATMENT FOR CANCER

Copyright ( 2007 by World Scientific Publishing Co. Pte. Ltd.

All rights reserved. This book, or parts thereof, may not be reproduced in any form or by any means, electronic or mechanical, including photocopying, recording or any information storage and retrieval system now known or to be invented, without written permission from the Publisher.
\end{abstract}

For photocopying of material in this volume, please pay a copying fee through the Copyright Clearance Center, Inc., 222 Rosewood Drive, Danvers, MA 01923, USA. In this case permission to photocopy is not required from the publisher.

ISBN-13 978-981-270-929-5

ISBN-10 981-270-929-0

Printed in Singapore. 
Editorial Board of the Annals of Traditional Chinese Medicine

\author{
Special Advisors: \\ Ke-Ji Chen (China) \\ Seung-Hoon Choi (Philippines) \\ David Eisenberg (USA) \\ Shi-Long Lai (Hong Kong) \\ Shichen Zhang (Hong Kong) \\ Xiaorui Zhang (Switzerland) \\ Chief Editors: \\ Ping-Chung Leung (Hong Kong) \\ Harry H.-S. Fong (USA) \\ Charlie Changli Xue (Australia) \\ Executive Editors: \\ William King-Fai Cheng (Hong Kong) \\ Sim-Kim Cheng (Singapore) \\ Chye-Tee Goh (Singapore)
}

\title{
Associate Editors:
}

Alan Bensoussan (Australia)

Ji-Sheng Han (China)

Paul Pui-Hay But (Hong Kong)

Joseph Tak-Fai Lau (Hong Kong)

Bao-Cang Cai (China)

Chun-Guang Li (Australia)

Kelvin K.-C. Chan (Hong Kong)

Liang Liu (Hong Kong)

Timothy M. Chan (USA)

David Story (Australia)

Pui-Kwong Chan (USA)

Frank Thien (Australia)

Wai-Yee Chan (USA)

Ling-Ling Wang (China)

Il-Moo Chang (Korea)

Kenji Watanabe (Japan)

Yung-Hsien Chang (Taiwan)

Kin-Ping Wong (USA)

Chun-Tao Che (Hong Kong)

Peishan Xie (China)

Chieh-Fu Chen (Taiwan)

Ping Xu (China)

Yung-Chi Cheng (USA)

Bing Zhang (China)

Moses Sing-Sum Chow (Hong Kong)

Zhong-Zhen Zhao (Hong Kong)

Kwok-Pui Fung (Hong Kong) 
This page intentionally left blank 


\section{Contents}

Contributors $\quad x i$

Preface to Series $\quad$ xvii

Preface to Volume $3 \quad$ xix

Chapter 1 The Scientific Basis of Chinese Medicine and 1

Cancer Care: A Western Medicine Perspective Stephen M. Sagar \& Raimond Wong

Chapter 2 Recent Status and Outlook of Traditional Chinese 55 Medicine in Cancer Treatment Dai-Han Zhou

Chapter 3 Chinese Medicine and Cancer Treatment in Hong Kong: A General Review Ping-Chung Leung, Vincent Ooi, Eliza L.-Y. Wong, Wai-Chun Au, Chun-Kwok Wong, Wai-Kei Lam, Sing-Fai Leung \& Tony S.-K. Mok

Chapter 4 Advancements of Ayurveda in Cancer Management with Special Focus on Hepatocellular Carcinoma Premalatha Balachandran

Chapter 5 Complementary Approaches to Cancer in Italy Ralph W. Moss 
Chapter 6 Kampo Treatment for Cancer

Kenji Watanabe

Chapter 7 Risk Management of Complementary Alternative Medicines in Cancer

Ursula Werneke

Chapter 8 Complementary Therapies for Cancer Patients

Barrie R. Cassileth, Jyothirmai Gubili \&

K. Simon Yeung

Chapter 9 Positive Findings about Herbs and Natural

Products Action on Cancer

Muriel J. Montbriand

Chapter 10 Mechanistic Studies on Combination of

Phytochemicals and Synthetic Drugs as

Anti-Cancer Agents

Shanmugam HemaIswarya \& Mukesh Doble

Chapter 11 Ethnopharmacology Approaches for Botanical

Immunomodulators and Chemoprotectants in Cancer Therapy

Patwardhan Bhushan \& Gautam Manish

Chapter 12 Bioactive Polysaccharides from TCM Herbs as Anti-Cancer Adjuvants

Raymond Chang

Chapter 13 Clinical Evaluation of Herbal Formula Decoction in Treating Non-Small Cell Lung Cancer by Various Rating Scales

Jie You \& Zhi-Ming Shi

Chapter 14 New Approach for Evaluating the Anti-Breast Cancer Activity of Traditional Chinese Medicine John M. Pezzuto, Richard C. Moon, Charles K.-H. Chang \& Ching-Jer Chang 
Chapter 15 Functional Magnetic Resonance Imaging Studies of Acupuncture

Gary Deng \& Barrie Cassileth

Index 
This page intentionally left blank 


\section{Contributors}

\section{Wai-Chun Au}

Centre for Clinical Trials on Chinese Medicine

The Institute of Chinese Medicine

School of Public Health

The Chinese University of Hong Kong

Shatin, New Territories, Hong Kong, SAR China

\section{Premalatha Balachandran}

National Center for Natural Products Research

Research Institute of Pharmaceutical Sciences, School of Pharmacy

University of Mississippi

University, MS 38677, USA

\section{Patwardhan Bhushan}

Interdisciplinary School of Health Sciences

University of Pune

Pune 411 007, India

\section{Barrie R. Cassileth}

Integrative Medicine Service

Memorial Sloan-Kettering Cancer Center

New York, NY 10021, USA

\section{Charles K.-H. Chang}

Mintong Pharmaceutical Company, Taichung, Taiwan 


\section{Ching-Jer Chang}

Department of Medicinal Chemistry and Molecular Pharmacology

School of Pharmacy and Pharmaceutical Sciences

Purdue University

West Lafayette, IN 47907-2091, USA

\section{Raymond Chang}

Institute of East-West Medicine

New York, NY 10016, USA

\section{Gary Deng}

Integrative Medicine Service

Memorial Sloan-Kettering Cancer Center

New York, NY 10021, USA

\section{Mukesh Doble}

Department of Biotechnology

Indian Institute of Technology Madras

Chennai-600036, India

\section{Jyothirmai Gubili}

Integrative Medicine Service

Memorial Sloan-Kettering Cancer Center

New York, NY 10021, USA

\section{Shanmugam HemaIswarya}

Department of Biotechnology

Indian Institute of Technology Madras

Chennai-600036, India

\section{Wai-Kei Lam}

Department of Chemical Pathology

Faculty of Medicine

Prince of Wales Hospital

The Chinese University of Hong Kong

Shatin, New Territories, Hong Kong, SAR China 


\section{Ping-Chung Leung}

Centre for Clinical Trials on Chinese Medicine

The Institute of Chinese Medicine

School of Public Health

The Chinese University of Hong Kong

Shatin, New Territories, Hong Kong, SAR China

\section{Sing-Fai Leung}

Department of Clinical Oncology

Faculty of Medicine

Prince of Wales Hospital

The Chinese University of Hong Kong

Shatin, New Territories, Hong Kong, SAR China

\section{Gautam Manish}

Interdisciplinary School of Health Sciences

University of Pune

Pune 411 007, India

\section{Tony S.-K. Mok}

Department of Clinical Oncology

Faculty of Medicine

Prince of Wales Hospital

The Chinese University of Hong Kong

Shatin, New Territories, Hong Kong, SAR China

\section{Muriel J. Montbriand}

Applied Research/Psychiatry

College of Medicine

University of Saskatchewan

Royal University Hospital

Saskatoon, SK, Canada S7N 0W8

\section{Richard C. Moon}

Department of Medicinal Chemistry and Molecular Pharmacology

School of Pharmacy and Pharmaceutical Sciences

Purdue University

West Lafayette, IN 47907-2091, USA 


\section{Ralph W. Moss}

Cancer Communications, Inc.

PO Box 1076

Lemont, PA 16851, USA

\section{Vincent Ooi}

Department of Biology

Faculty of Science

The Chinese University of Hong Kong

Shatin, New Territories, Hong Kong, SAR China

\section{John M. Pezzuto}

Department of Medicinal Chemistry and Molecular Pharmacology

School of Pharmacy and Pharmaceutical Sciences

Purdue University

West Lafayette, IN 47907-2091, USA

Department of Pharmaceutical Sciences, College of Pharmacy

University of Hawaii at Hilo

Hilo, HI 96720, USA

\section{Stephen M. Sagar}

Departments of Oncology and Medicine

McMaster University

Juravinski Cancer Centre

Hamilton, ON, Canada L8V 5C2

\section{Zhi-Ming Shi}

Tumor Department

Clinical Oncology Centre

Longhua Hospital Affiliated to

Shanghai University of Traditional Chinese Medicine Shanghai 200036, P. R. China

\section{Kenji Watanabe}

Department of Kampo Medicine

Keio University School of Medicine

Tokyo 160-8582, Japan 


\section{Ursula Werneke}

Department of Psychiatry

Homerton University Hospital

London E9 6SR, UK

\section{Chun-Kwok Wong}

Department of Chemical Pathology

Faculty of Medicine

Prince of Wales Hospital

The Chinese University of Hong Kong

Shatin, New Territories, Hong Kong, SAR China

\section{Eliza L.-Y. Wong}

Department of Community and Family Medicine

School of Public Health

Faculty of Medicine

Prince of Wales Hospital

The Chinese University of Hong Kong

Shatin, New Territories, Hong Kong, SAR China

\section{Raimond Wong}

Departments of Oncology and Medicine

McMaster University

Juravinski Cancer Centre

Hamilton, ON, Canada L8V 5C2

\section{K. Simon Yeung}

Integrative Medicine Service

Memorial Sloan-Kettering Cancer Center

New York, NY 10021, USA

\section{Jie You}

Tumor Department

Clinical Oncology Centre

Longhua Hospital Affiliated to

Shanghai University of Traditional Chinese Medicine Shanghai 200036, P. R. China 


\section{Dai-Han Zhou}

Cancer Centre, No. 1 Affiliated Hospital

Chinese Medicine University

Guangzhou 510405, P. R. China 


\section{Preface to Series}

\section{Does Traditional Chinese Medicine Work?}

History should be acknowledged and respected. Despite this, the historical value of Chinese medicine in China and some parts of Asia should not be used as the only important evidence of efficacy.

While clinical science has followed closely the principles of deductive research in science and developed its methodology of wide acceptance, there is a natural demand from both users and service providers that the same methodology be applied to the traditional art of healing. There should be only one scale for the measurement of efficacy. Thus, evidencebased medicine, which apparently is the only acceptable form of treatment, would also claim its sovereignty in Chinese medicine.

In spite of influential proponents and diligent practitioners, efforts relating to the application of evidence-based medicine methodology to Chinese medicine research have been slow and unimpressive. This should not come as a surprise. Evidence-based medicine requires the knowledge of the exact chemistry of the drug used, the exact physical or chemical activities involved and above all, the biological responses in the recipient. All these are not known. Working back from the black box of old historical records of efficacy requires huge resources and time, if at all possible. Insistence on this approach would result in either unending frustrations or utter desperation.

Parallel with the modern attempts, respectable Chinese medicine practitioners have unendingly and relentlessly cried out their objection to the evidence-based approach. They insisted that all the evidences were already there from the Classical Records. Forcing the classical applications through a rigid modern framework of scrutiny is artificially coating Chinese medicine with a scientific clothing that does not fit. 
Thus, the modern proponents are facing an impasse when they rely totally on modern scientific concepts. The traditional converts are persisting to push their pilgrims of defense. Where do we stand so as to achieve the best results of harmonisation?

There must be a compromise somewhere. Classic evidences can be transformed into a universal language to be fairly evaluated and to be decided whether suitable for further research, using the deductive methodology or an innovative one after intelligent modifications.

There is a need for a platform on which a direction can be developed in the attempt to modernise the traditional art and science of healing, while remaining free and objective to utilise the decaying wisdom without prejudice.

With the growing demand for complementary/alternative medicine from the global public and a parallel interest from the service providers, there is an urgent need for the provision of valuable information in this area.

The Annals of Chinese Medicine is a timely serial publication responding to this need. It will be providing authoritative and current information about Chinese medicine in the areas of clinical trials, biological activities of herbs, education, research and quality control requirements. Contributors are invited to send in their reports and reviews to ensure quality and value. Clinicians and scientists who are willing to submit their valuable observations, resulting from their painstaking researches are welcome to send in their manuscripts. The Annals of Chinese Medicine has the objective of providing a lasting platform for all who concentrate their efforts on the modernization of Chinese medicine.

Professor Ping-Chung Leung Institute of Chinese Medicine The Chinese University of Hong Kong 


\section{Preface to Volume 3}

The third volume of the Annals of Traditional Chinese Medicine carries the theme of Cancer Treatment. Since the commencement of this book series, the Editorial Board has emphasized its main objective, that is to help modernize Chinese medicine, thus providing a convenient platform for all scientists, including those who belong to the traditional camp as well as members of the modern scientific community who insist on strict deductive approaches.

Cancer is obviously one of the most common areas where demand for alternative treatment is overwhelming. A significant portion of the American and European populations regularly consumes health supplements to either prevent cancer or as adjuvant therapy during cancer treatment. Health surveys conducted in Hong Kong (a city of predominantly Chinese people) have shown that over $90 \%$ of cancer patients consume products of Chinese medicine, either prescribed by Chinese medicine practitioners or purchased over-the-counter. Choosing Cancer as the theme of our third volume is therefore appropriate. There is no intention of guiding the reader on the choice of therapy, nor is there any suggestion of preferences of the available options of treatment (although a few solid examples are given). Instead we want to introduce the justification and logic of using Chinese medicine as an adjunct, discuss about the basic principles and the options that are available, expose the regional and cultural varieties, and some problems related to their use, describe some natural products of particular interests and lastly, give examples of specific cancers being treated with herbal therapy.

Using modern scientific concepts to understand and explain traditional or alternative medicine is logical and unavoidable. Indeed, the Annals has already adopted this principle. Nevertheless, we welcome distinguished 
scholars of the very traditional stream to explain their views, particularly at this time, in the popular field of cancer.

Professor Dai-Han Zhou is a learned scholar in Chinese medicine who has written extensively on the principles and practice of Chinese medicine for cancer patients. Readers will find his articles quite outof-the-norm as he stresses on holistic care and the importance of individualized therapy. On the other hand, Prof. Zhou tries to equate herbalists' observations as evidence-based medicine, and assumes that some herbs work via specific channels resembling those being targeted by new therapies like Iressa, Tarceva and Avastin. Professor Zhou quoted one clinical trial using a proprietor herbal medicine. In that situation, he accepts generalization and ignores individualization. The article is really interesting because it exposes the complexity of thoughts of the modern day Chinese medicine expert. While the discrepancies observed may be controversial, Prof. Zhou's strong criticisms against over-treatment in modern medicine and his call for higher rates of survival among cancer patients should be established as good advice for all.

Professor Zhou's orthodox account is well balanced with Professor Stephen Sagar's Western medicine perspectives on the scientific basis of cancer care in Chinese medicine.

This volume attempts to give very rich accounts of the varieties of cancer treatment available, as well as the cultural differences in China, Hong Kong, Japan, India, Italy, the United Kingdom and the United States. There are altogether seven chapters sharing this load.

Since herbal medicine can be the main basis for the maintenance of survival in spite of the persistence of cancer or cancer metastases, natural products and phytochemistry that contribute toward survival via various channels should be explored. This volume provides a number of high quality articles (by authors from Canada, India and the US) on the subject. Lastly, there are some real examples of integrated treatment for specific cancers that will be of interest to readers. Two good examples from China are chosen.

With the growing demand for complementary and alternative medicine from the global public and a parallel interest from the service providers, we believe that this volume will provide valuable information in the area of cancer for all. 